\title{
Irrationality of certain numbers that contain values of the di- and trilogarithm
}

Kh. Hessami Pilehrood* (Shahrekord), T. Hessami Pilehrood ${ }^{\dagger}$ (Shahrekord) and W. Zudilin ${ }^{\ddagger}$ (Moscow)

October 26, 2012

\section{Abstract}

We prove that, for $z \in\{1 / 2,2 / 3,3 / 4,4 / 5\}$, at least one of the two numbers

$$
\sum_{l=1}^{\infty} \frac{z^{l}}{l^{2}}+\log (1-z) \log z \quad \text { and } \quad \sum_{l=1}^{\infty} \frac{z^{l}}{l^{3}}+\frac{1}{2} \log (1-z) \log ^{2} z
$$

is irrational.

The irrationality result that we prove here, involves the values of the functions

$$
f_{j}(z)=\operatorname{Li}_{j}(z)-\operatorname{Li}_{1}(z) \cdot \frac{\log ^{j-1} z}{(j-1) !}, \quad j=2,3, \ldots
$$

Here, as usual,

$$
\operatorname{Li}_{j}(z)=\sum_{l=1}^{\infty} \frac{z^{l}}{l^{j}}, \quad|z|<1, \quad j=1,2,3, \ldots,
$$

denote the polylogarithmic functions; $\operatorname{Li}_{1}(z)=-\log (1-z)$.

Theorem 1. For $z \in\{1 / 2,2 / 3,3 / 4,4 / 5\}$, at least one of the two numbers $f_{2}(z)$ and $f_{3}(z)$ is irrational.

${ }^{*}$ Kh. Hessami Pilehrood was supported in part by Institute for Studies in Theoretical Physics and Mathematics (IPM) grant no. 83110021.

${ }^{\dagger}$ T. Hessami Pilehrood was supported in part by IPM grant no. 83110020.

${ }^{\ddagger}$ W. Zudilin was supported in part by Russian Foundation for Basic Research grant no. 03-0100359 . 
Using the classical formulae (see, e.g., [5], eqs. (1.11) and (6.10)) we may also express the functions $f_{2}(z)$ and $f_{3}(z)$ as follows:

$$
\begin{gathered}
f_{2}(z)=\frac{\pi^{2}}{6}-\operatorname{Li}_{2}(1-z), \\
f_{3}(z)=\zeta(3)+\frac{1}{6} \log ^{3} z+\frac{\pi^{2}}{6} \log z-\operatorname{Li}_{3}(1-z)-\operatorname{Li}_{3}\left(1-z^{-1}\right) .
\end{gathered}
$$

Moreover, the values of these functions at the point $z=1 / 2$ are computed by means of $\log 2, \zeta(2)=\pi^{2} / 6$ and $\zeta(3)$ (see [5], eqs. (1.16) and (6.12)):

$$
f_{2}(1 / 2)=\frac{1}{2} \zeta(2)+\frac{1}{2} \log ^{2} 2, \quad f_{3}(1 / 2)=\frac{7}{8} \zeta(3)-\frac{1}{2} \zeta(2) \log 2-\frac{1}{3} \log ^{3} 2 .
$$

Using these formulae we obtain the following corollary of Theorem 1.

Theorem 2. At least one of the two numbers

$$
\pi^{2}+6 \log ^{2} 2 \quad \text { and } \quad \zeta(3)-\frac{2}{21}\left(\pi^{2}+4 \log ^{2} 2\right) \log 2
$$

is irrational.

Relative results, 'at least one of the numbers $3 \zeta(3)-c \zeta(2), \zeta(2)-2 c \log 2(c \in \mathbb{Q})$ is irrational' and 'at least one of the numbers $\operatorname{Li}_{2}(1 / q), \operatorname{Li}_{3}(1 / q)(q \in \mathbb{Z} \backslash\{0,2\})$ is irrational', are proved in [3] and [4], respectively.

Our proof relies on a general hypergeometric construction of two linear forms in the polylogarithms and positive powers of the logarithm, respectively. This idea was recently used in [2] for proving that at least one of the three numbers $f_{2}(1 / 2)$, $f_{3}(1 / 2)$ and $f_{4}(1 / 2)$ is irrational. We are able to improve this earlier result and present the related ones due to the powerful group-structure arithmetic method introduced in [6] and [7] in order to prove new estimates for irrationality measures of $\zeta(2)$ and $\zeta(3)$ (see also [8] and [9]).

\section{Hypergeometric series and integral}

Let $a_{0}, a_{1}, \ldots, a_{s}$ and $b_{0}, b_{1}, \ldots, b_{s}$ be collections of positive integers satisfying

$$
\begin{gathered}
b_{0}=1<a_{0}, a_{1}, \ldots, a_{s}<b_{1}, \ldots, b_{s}, \quad \sum_{j=0}^{s} a_{j}<\sum_{j=0}^{s} b_{j}, \\
\min _{0 \leqslant j \leqslant s}\left\{a_{j}\right\}+\max _{0 \leqslant j \leqslant s}\left\{a_{j}\right\} \leqslant b_{l} \quad \text { for all } l=1, \ldots, s,
\end{gathered}
$$

We will also consider the ordered versions $\left\{a_{0}^{*}, a_{1}^{*}, \ldots, a_{s}^{*}\right\}=\left\{a_{0}, a_{1}, \ldots, a_{s}\right\}$ and $\left\{b_{0}^{*}, b_{1}^{*}, \ldots, b_{s}^{*}\right\}=\left\{b_{0}, b_{1}, \ldots, b_{s}\right\}$ of the collections:

$$
a_{0}^{*} \leqslant a_{1}^{*} \leqslant \ldots \leqslant a_{s}^{*}, \quad b_{0}^{*}=1, \quad b_{1}^{*} \geqslant b_{2}^{*} \geqslant \ldots \geqslant b_{s}^{*} .
$$


To this data we assign the rational function

$$
\widetilde{R}(t)=\widetilde{R}(\boldsymbol{a}, \boldsymbol{b} ; t)=\prod_{j=0}^{s} \frac{\Gamma\left(t+a_{j}\right)}{\Gamma\left(t+b_{j}\right)}=\frac{(t+1)(t+2) \cdots\left(t+a_{0}-1\right)}{\prod_{j=1}^{s}\left(t+a_{j}\right) \cdots\left(t+b_{j}-1\right)}
$$

and its 'arithmetically normalized' version

$$
R(t)=R(\boldsymbol{a}, \boldsymbol{b} ; t)=\frac{\prod_{j=1}^{s} \Gamma\left(b_{j}-a_{j}\right)}{\Gamma\left(a_{0}\right)} \widetilde{R}(\boldsymbol{a}, \boldsymbol{b} ; t)=\sum_{j=1}^{s} \sum_{k=a_{j}^{*}}^{b_{j}^{*}-1} \frac{A_{j k}}{(t+k)^{j}} .
$$

Our main objects then are as follows:

$$
\begin{aligned}
I & =I(\boldsymbol{a}, \boldsymbol{b} ; z)=z^{a_{0}^{*}} \sum_{t=0}^{\infty} R(t) z^{t}=\sum_{t=1-a_{0}^{*}}^{\infty} R(t) z^{t+a_{0}^{*}}=\sum_{j=1}^{s} \sum_{k=a_{j}^{*}}^{b_{j}^{*}-1} A_{j k} z^{-k+a_{0}^{*}} \sum_{t=1-a_{0}^{*}}^{\infty} \frac{z^{t+k}}{(t+k)^{j}} \\
& =\sum_{j=1}^{s} \sum_{k=a_{j}^{*}}^{b_{j}^{*}-1} A_{j k} z^{-k+a_{0}^{*}} \sum_{l=1+k-a_{0}^{*}}^{\infty} \frac{z^{l}}{l^{j}}=\sum_{j=1}^{s} \sum_{k=a_{j}^{*}}^{b_{j}^{*}-1} A_{j k} z^{-k+a_{0}^{*}}\left(\operatorname{Li}_{j}(z)-\sum_{l=1}^{k-a_{0}^{*}} \frac{z^{l}}{l^{j}}\right) \\
& =\sum_{j=1}^{s} \operatorname{Li}_{j}(z) \cdot \sum_{k=a_{j}^{*}} A_{j k} z^{-k+a_{0}^{*}}-\sum_{j=1}^{s} \sum_{k=a_{j}^{*}}^{b_{j}^{*}-1} A_{j k} \sum_{l=1}^{k-a_{0}^{*}} \frac{z^{-\left(k-a_{0}^{*}-l\right)}}{l^{j}} \\
& =\sum_{j=1}^{s} P_{j}\left(z^{-1}\right) \operatorname{Li}_{j}(z)-P_{0}\left(z^{-1}\right),
\end{aligned}
$$

and (the closed contour $\mathcal{L}$ below surrounds all poles $t=-k$ for $a_{1}^{*} \leqslant k<b_{1}^{*}$ of the rational function $R(t)$ )

$$
\begin{aligned}
J & =J(\boldsymbol{a}, \boldsymbol{b} ; z)=\frac{z^{a_{0}^{*}}}{2 \pi i} \oint_{\mathcal{L}} R(t) z^{t} \mathrm{~d} t=z^{a_{0}^{*}} \sum_{k} \operatorname{Res}_{t=-k}\left(R(t) z^{t}\right) \\
& =\sum_{k} z^{-k+a_{0}^{*}} \operatorname{Res}_{t=-k}\left(R(t) z^{t+k}\right)=\sum_{j=1}^{s} \sum_{k} A_{j k} z^{-k+a_{0}^{*}} \cdot \operatorname{Res}_{t=-k} \frac{z^{t+k}}{(t+k)^{j}} \\
& =\sum_{j=1}^{s} \sum_{k} A_{j k} z^{-k+a_{0}^{*}} \cdot \operatorname{Res}_{t=0} \frac{z^{t}}{t^{j}}=\sum_{j=1}^{s} \sum_{k} A_{j k} z^{-k+a_{0}^{*}} \cdot \frac{\log ^{j-1} z}{(j-1) !} \\
& =\sum_{j=1}^{s} P_{j}\left(z^{-1}\right) \frac{\log ^{j-1} z}{(j-1) !} .
\end{aligned}
$$

All this means that we arrange to construct 'simultaneous' approximations to the set of polylogarithms $\operatorname{Li}_{1}(z), \ldots, \operatorname{Li}_{s}(z)$ and the set of logarithm powers $\log z, \ldots$, $\frac{1}{(s-1) !} \log ^{s-1} z$. This is essentially the idea from [2], Theorem 3 . 


\section{Arithmetic ingredients}

Lemma 1. Let

$$
m_{1}=\max \left\{a_{0}-1, \max _{1 \leqslant j \leqslant s}\left\{b_{j}\right\}-\min _{1 \leqslant j \leqslant s}\left\{a_{j}\right\}-1\right\} .
$$

Then, for any $j=1,2, \ldots, s$, we have the inclusions

$$
D_{m_{1}}^{s-j} \cdot A_{j k} \in \mathbb{Z}
$$

where $A_{j k}$ are the coefficients in the partial-fraction decomposition (3).

Proof. The rational function in (3) may be written as $R(t)=\prod_{j=0}^{s} R_{j}(s)$, where

$$
R_{0}(t)=\frac{(t+1)(t+2) \cdots\left(t+a_{0}-1\right)}{\left(a_{0}-1\right) !}
$$

and

$$
R_{j}(t)=\frac{\left(b_{j}-a_{j}-1\right) !}{\left(t+a_{j}\right) \cdots\left(t+b_{j}-1\right)} \quad \text { for } j=1, \ldots, s .
$$

The polynomial $R_{0}(t)$ (of degree $a_{0}-1$ ) is integer-valued, with the property

$$
\left.D_{a_{0}-1}^{l} \cdot \frac{1}{l !} \frac{\mathrm{d}^{l} R_{0}(t)}{\mathrm{d} t^{l}}\right|_{t=-k} \in \mathbb{Z}, \quad k \in \mathbb{Z}, \quad l=0,1,2, \ldots
$$

(see [9], Lemma 15). All poles of the rational functions $R_{1}(t), \ldots, R_{s}(t)$ are of the form $t=-k$ for some integer $k$ in the range $a^{\prime} \leqslant k<b^{\prime}$, where $a^{\prime}=\min _{1 \leqslant j \leqslant s}\left\{a_{j}\right\}$ and $b^{\prime}=\max _{1 \leqslant j \leqslant s}\left\{b_{j}\right\}$. Therefore, for any $j=1, \ldots, s$, we have

$$
\left.D_{b^{\prime}-a^{\prime}-1}^{l} \cdot \frac{1}{l !} \frac{\mathrm{d}^{l}}{\mathrm{~d} t^{l}}\left(R_{j}(t)(t+k)\right)\right|_{t=-k} \in \mathbb{Z}, \quad k=a^{\prime}, a^{\prime}+1, \ldots, b^{\prime}-1, \quad l=0,1,2, \ldots
$$

(see [9], Lemma 16). Finally, by the Leibniz rule

$$
\begin{aligned}
A_{s-l, k}= & \left.\frac{1}{l !} \frac{\mathrm{d}^{l}}{\mathrm{~d} t^{l}}\left(R(t)(t+k)^{s}\right)\right|_{t=-k} \\
= & \left.\left.\sum_{\substack{l_{0}, l_{1}, \ldots, l_{s} \geqslant 0 \\
l_{0}+l_{1}+\cdots+l_{s}=l}} \frac{1}{l_{0} !} \frac{\mathrm{d}^{l_{0}} R_{0}(t)}{\mathrm{d} t^{l_{0}}}\right|_{t=-k} \cdot \frac{1}{l_{1} !} \frac{\mathrm{d}^{l_{1}}}{\mathrm{~d} t^{l_{1}}}\left(R_{1}(t)(t+k)\right)\right|_{t=-k} \ldots \\
& \times\left.\frac{1}{l_{s} !} \frac{\mathrm{d}^{l_{s}}}{\mathrm{~d} t^{l_{s}}}\left(R_{s}(t)(t+k)\right)\right|_{t=-k}
\end{aligned}
$$

for any $k=a^{\prime}, a^{\prime}+1, \ldots, b^{\prime}-1$ and $l=0,1, \ldots, s-1$. In view of inclusions (7) and (8), the last formula yields the required result. 
Lemma 2. Let $m=\max \left\{m_{1}, b_{2}^{*}-a_{0}^{*}-1\right\}$ with $m_{1}$ defined in (6) and

$$
m_{0}=b_{1}^{*}-a_{0}^{*}-1=\max _{1 \leqslant j \leqslant s}\left\{b_{j}\right\}-\min _{0 \leqslant j \leqslant s}\left\{a_{j}\right\}-1 .
$$

Then the polynomials

$$
P_{j}(x)=\sum_{k=a_{j}^{*}}^{b_{j}^{*}-1} A_{j k} x^{k-a_{0}^{*}}, \quad j=1, \ldots, s, \quad P_{0}(x)=\sum_{j=1}^{s} \sum_{k=a_{j}^{*}}^{b_{j}^{*}-1} A_{j k} \sum_{l=1}^{k-a_{0}^{*}} \frac{x^{k-a_{0}^{*}-l}}{l^{j}},
$$

defined in (4) and (5) as the coefficients of linear forms satisfy

$$
\operatorname{deg} P_{j}(x) \leqslant m_{0}, \quad D_{m_{0}} D_{m}^{s-1} \cdot P_{j}(x) \in \mathbb{Z}[x], \quad j=0,1, \ldots, s .
$$

Proof. The claim follows immediately for the polynomials $P_{1}(x), \ldots, P_{s}(x)$ from Lemma 1 since $m \geqslant m_{1}$. In the case of $P_{0}(x)$ we require the inclusions of Lemma 1 , also the obvious ones

$$
D_{b_{1}^{*}-a_{0}^{*}-1} \cdots D_{b_{j}^{*}-a_{0}^{*}-1} \cdot \sum_{l=1}^{k-a_{0}^{*}} \frac{x^{k-a_{0}^{*}-l}}{l^{j}} \in \mathbb{Z}[x], \quad k=a_{j}^{*}, \ldots, b_{j}^{*}-1, \quad j=1, \ldots, s,
$$

and the fact that $m \geqslant b_{j}^{*}-a_{0}^{*}-1$ for $j=2, \ldots, s$.

Denote by

$$
\Pi=\Pi(\boldsymbol{a}, \boldsymbol{b})=\frac{\prod_{j=1}^{s} \Gamma\left(b_{j}-a_{j}\right)}{\Gamma\left(a_{0}\right)}=\frac{\prod_{j=1}^{s}\left(b_{j}-a_{j}-1\right) !}{\left(a_{0}-1\right) !}
$$

the arithmetic normalization in (3). By (2)-(5), the quantities

$$
\frac{I(\boldsymbol{a}, \boldsymbol{b} ; z)}{\Pi(\boldsymbol{a}, \boldsymbol{b})} \quad \text { and } \quad \frac{J(\boldsymbol{a}, \boldsymbol{b} ; z)}{\Pi(\boldsymbol{a}, \boldsymbol{b})}
$$

do not depend on rearranging the parameters $a_{0}, a_{1}, \ldots, a_{s}$. Let us denote by $\mathfrak{S}$ the permutation group (of order $(s+1)$ !) of the parameters $a_{0}, a_{1}, \ldots, a_{s}$. For any $\sigma \in \mathfrak{S}$, introduce the corresponding normalization factor $\Pi^{(\sigma)}=\Pi(\sigma \boldsymbol{a}, \boldsymbol{b})$ and the quantities

$$
\begin{aligned}
& I^{(\sigma)}=I(\sigma \boldsymbol{a}, \boldsymbol{b} ; z)=\sum_{j=1}^{s} P_{j}^{(\sigma)}\left(z^{-1}\right) \operatorname{Li}_{j}(z)-P_{0}^{(\sigma)}\left(z^{-1}\right), \\
& J^{(\sigma)}=J(\sigma \boldsymbol{a}, \boldsymbol{b} ; z)=\sum_{j=1}^{s} P_{j}^{(\sigma)}\left(z^{-1}\right) \frac{\log ^{j-1} z}{(j-1) !}
\end{aligned}
$$

Using Lemma 2 , the inequality $m_{0} \geqslant m$, and invariance of $(10)$ and $m_{0}=b_{1}^{*}-a_{0}^{*}-1$ under the $\mathfrak{S}$-action we arrive at the following claim. 
Lemma 3. The following inclusions

$$
D_{m_{0}}^{s} \cdot \frac{\Pi^{(\sigma)}}{\Pi} \cdot P_{j}(x)=D_{m_{0}}^{s} \cdot P_{j}^{(\sigma)}(x) \in \mathbb{Z}[x], \quad j=0,1, \ldots, s,
$$

are valid for any $\sigma \in \mathfrak{S}$.

Corollary (cf. [9], Lemma 10). If

$$
\Phi=\Phi(\boldsymbol{a}, \boldsymbol{b})=\prod_{\sqrt{m_{0}}<p \leqslant m} p^{e_{p}}, \quad \text { where } \quad e_{p}=\max _{\sigma \in \mathfrak{S}} \operatorname{ord}_{p} \frac{\Pi}{\Pi^{(\sigma)}},
$$

then

$$
D_{m_{0}} D_{m}^{s-1} \cdot \Phi^{-1} \cdot P_{j}(x) \in \mathbb{Z}[x], \quad j=0,1, \ldots, s .
$$

Since $\operatorname{ord}_{p} N !=\lfloor N / p\rfloor$ for primes $p>\sqrt{N}$, we have

$$
\operatorname{ord}_{p} \Pi(\boldsymbol{a}, \boldsymbol{b})=\sum_{j=1}^{s}\left\lfloor\frac{b_{j}-a_{j}-1}{p}\right\rfloor-\left\lfloor\frac{a_{0}-1}{p}\right\rfloor, \quad p>\sqrt{m_{0}},
$$

hence

$$
e_{p}=\max _{\sigma \in \mathfrak{S}} \varpi_{p}^{(\sigma)}
$$

where

$$
\varpi_{p}^{(\sigma)}=\sum_{j=1}^{s}\left(\left\lfloor\frac{b_{j}-a_{j}-1}{p}\right\rfloor-\left\lfloor\frac{b_{j}-\sigma a_{j}-1}{p}\right\rfloor\right)-\left(\left\lfloor\frac{a_{0}-1}{p}\right\rfloor-\left\lfloor\frac{\sigma a_{0}-1}{p}\right\rfloor\right) .
$$

Now, take the parameters $\boldsymbol{a}, \boldsymbol{b}$ as follows:

$$
a_{j}=\alpha_{j} n+1, \quad j=0,1, \ldots, n, \quad \text { and } \quad b_{j}=\beta_{j} n+2, \quad j=1, \ldots, n,
$$

where $\alpha_{0}, \alpha_{1}, \ldots, \alpha_{n}$ and $\beta_{1}, \ldots, \beta_{n}$ are positive integers satisfying

$$
\begin{gathered}
0<\alpha_{0}, \alpha_{1}, \ldots, \alpha_{s}<\beta_{1}, \ldots, \beta_{s}, \quad \sum_{j=0}^{s} \alpha_{j}<\sum_{j=1}^{s} \beta_{j}, \\
\min _{0 \leqslant j \leqslant s}\left\{\alpha_{j}\right\}+\max _{0 \leqslant j \leqslant s}\left\{\alpha_{j}\right\} \leqslant \beta_{l} \quad \text { for all } l=1, \ldots, s,
\end{gathered}
$$

to ensure conditions (1). As before, we introduce the ordered collections

$$
\begin{gathered}
\left\{\alpha_{0}^{*}, \alpha_{1}^{*}, \ldots, \alpha_{s}^{*}\right\}=\left\{\alpha_{0}, \alpha_{1}, \ldots, \alpha_{s}\right\}, \quad\left\{\beta_{1}^{*}, \ldots, \beta_{s}^{*}\right\}=\left\{\beta_{1}, \ldots, \beta_{s}\right\}, \\
\alpha_{0}^{*} \leqslant \alpha_{1}^{*} \leqslant \ldots \leqslant \alpha_{s}^{*}, \quad \beta_{1}^{*} \geqslant \ldots \geqslant \beta_{s}^{*} .
\end{gathered}
$$

Then $m_{0}=\nu_{0} n, m=\nu n$, where

$$
\begin{gathered}
\nu_{0}=\beta_{1}^{*}-\alpha_{0}^{*}=\max _{1 \leqslant j \leqslant s}\left\{\beta_{j}\right\}-\min _{0 \leqslant j \leqslant s}\left\{\alpha_{j}\right\}, \\
\nu=\max \left\{\alpha_{0}, \max _{1 \leqslant j \leqslant s}\left\{\beta_{j}\right\}-\min _{1 \leqslant j \leqslant s}\left\{\alpha_{j}\right\}, \max _{2 \leqslant j \leqslant s}\left\{\beta_{j}\right\}-\min _{0 \leqslant j \leqslant s}\left\{\alpha_{j}\right\},\right.
\end{gathered}
$$


and

$$
\varpi_{p}^{(\sigma)}=\varphi^{(\sigma)}\left(\frac{n}{p}\right)
$$

where

$$
\varphi^{(\sigma)}(x)=\sum_{j=1}^{s}\left(\left\lfloor\left(\beta_{j}-\alpha_{j}\right) x\right\rfloor-\left\lfloor\left(\beta_{j}-\sigma \alpha_{j}\right) x\right\rfloor\right)-\left(\left\lfloor\alpha_{0} x\right\rfloor-\left\lfloor\sigma \alpha_{0} x\right\rfloor\right), \quad \sigma \in \mathfrak{S} .
$$

With a help of identity $\lfloor y\rfloor=y-\{y\}$ (where $\{\cdot\}$ denotes the fractional part of a number) we see that functions (14) are 1-periodic. Moreover, since $\lfloor k x\rfloor$ (for a positive integer $k$ ) changes its value only at the points $x=l / k$ for $l \in \mathbb{Z}$, we conclude that any of the functions in (14) takes constant values in demi-intervals $[u, v) \subset[0,1)$, where $u, v$ is any pair of neighbour fractions with denominators $\leqslant \nu_{0}$. These properties are inherited by the function

$$
\varphi(x)=\max _{\sigma \in \mathfrak{S}} \varphi^{(\sigma)}(x),
$$

whence its computation is a pure machinery.

Denoting $\Phi_{n}=\Phi(\boldsymbol{a}, \boldsymbol{b})$ and using the prime number theorem and the standard arithmetic argument (see, e.g., [9], Lemma 11) we obtain

Lemma 4. The following limit relations are valid:

$$
\begin{gathered}
\lim _{n \rightarrow \infty} \frac{\log \Phi_{n}}{n}=\int_{0}^{1} \varphi(x) \mathrm{d} \psi(x)-\int_{0}^{1 / \nu} \varphi(x) \frac{\mathrm{d} x}{x^{2}}, \\
\lim _{n \rightarrow \infty} \frac{\log D_{\nu_{0} n}}{n}=\nu_{0}, \quad \lim _{n \rightarrow \infty} \frac{\log D_{\nu n}}{n}=\nu,
\end{gathered}
$$

where $\psi(x)$ is the logarithmic derivative of the gamma function.

In accordance with (11), formulae (15) completely determine the arithmetic behaviour of the constructed approximations (4) and (5).

\section{Asymptotics}

Consider the integral

$$
J=J(\boldsymbol{a}, \boldsymbol{b} ; z)=\frac{z^{a_{0}^{*}}}{2 \pi i} \oint_{\mathcal{L}} R(t) z^{t} \mathrm{~d} t
$$

where $\mathcal{L}$ is a closed clockwise contour surrounding all poles $t=-k$ for $a_{1}^{*} \leqslant k<b_{1}^{*}$ of the rational function $R(t)=R(\boldsymbol{a}, \boldsymbol{b} ; t)$. 
Lemma 5. Let $0<z<1$ and $s \geqslant 1$. Then the following integral representation is valid:

$$
J=-\frac{z^{a_{0}^{*}}}{2 \pi i} \int_{M-i \infty}^{M+i \infty} R(t) z^{t} \mathrm{~d} t
$$

where $M$ is an arbitrary real constant in the interval $\left(-\infty,-b_{1}^{*}\right)$.

Proof. Take the contour $\mathcal{L}$ in (16) as the rectangle with vertices $M \pm i T, T \pm i T$, where $M$ is an arbitrary fixed number, $M \leqslant-b_{1}^{*}$, and $T$ is sufficiently large. In accordance with (2), (12) we have that $R(t)=o\left(T^{-s-1}\right)$ on the segments $[M-i T$, $T-i T],[T-i T, T+i T]$ and $[T+i T, M+i T]$. Therefore, for horizontal sides of the rectangle we obtain

$$
\left|\int_{M \pm i T}^{T \pm i T} R(t) z^{t} \mathrm{~d} t\right|=\left|\int_{M}^{T} R(x \pm i T) z^{x \pm i T} \mathrm{~d} x\right| \leqslant \frac{c_{1}}{T^{2}}|z|^{M}(T-M),
$$

while on the vertical segment $[T-i T, T+i T]$ we have the trivial bound $|z|^{t}=$ $\left|z^{T+i y}\right|=|z|^{T}<1$, hence

$$
\left|\int_{T-i T}^{T+i T} R(t) z^{t} \mathrm{~d} t\right| \leqslant \frac{c_{2}}{T}
$$

Finally, letting $T$ tend to infinity and taking into account estimates (18) and (19) we arrive at the required statement.

Lemma 6. The integral $J=J(\boldsymbol{a}, \boldsymbol{b} ; z)$ admits the following representation:

$$
J=c_{3} \int_{\mu-i \infty}^{\mu+i \infty} g(\tau) e^{n f(\tau)}\left(1+O\left(n^{-1}\right)\right) \mathrm{d} t
$$

where the functions

$$
\begin{aligned}
f(\tau)=\sum_{j=0}^{s} & \left(\left(\tau-\beta_{j}\right) \log \left(\tau-\beta_{j}\right)-\left(\tau-\alpha_{j}\right) \log \left(\tau-\alpha_{j}\right)\right) \\
& \quad-\alpha_{0} \log \alpha_{0}+\sum_{j=1}^{s}\left(\beta_{j}-\alpha_{j}\right) \log \left(\beta_{j}-\alpha_{j}\right)+\left(\alpha_{0}^{*}-\tau\right) \log z
\end{aligned}
$$

and

$$
g(\tau)=\exp \left\{\frac{1}{2} \sum_{j=0}^{s}\left(\log \left(\tau-\alpha_{j}\right)-3 \log \left(\tau-\beta_{j}\right)\right)\right\}
$$

are defined in the cut $\tau$-plane $\mathbb{C} \backslash\left(-\infty, \beta_{1}^{*}\right], \mu$ is an arbitrary number in the interval $\left(\beta_{1}^{*},+\infty\right)$, and the constants $c_{3} \neq 0$ and in $O(1 / n)$ depend only on the parameters $\boldsymbol{\alpha}, \boldsymbol{\beta}$. 
Proof. Changing the variable $t$ by $-t$ and applying the formula

$$
\Gamma(a-t) \Gamma(t-a+1)=\frac{\pi}{\sin (\pi(a-t))},
$$

we can write $(17)$ in the form

$$
J(\boldsymbol{a}, \boldsymbol{b} ; z)= \pm \frac{z^{a_{0}^{*}}}{2 \pi i} \frac{\prod_{j=1}^{s} \Gamma\left(b_{j}-a_{j}\right)}{\Gamma\left(a_{0}\right)} \int_{-M-i \infty}^{-M+i \infty} \prod_{j=0}^{s} \frac{\Gamma\left(t-b_{j}+1\right)}{\Gamma\left(t-a_{j}+1\right)} z^{-t} \mathrm{~d} t
$$

for some choice of the sign in ' \pm '. Set $-M=\mu n, t=n \tau$, where $\tau=\mu+i y$, $\mu \in\left(\beta_{1}^{*}, \infty\right), y \in(-\infty,+\infty)$, and $n \in \mathbb{N}$, use (12) and the following asymptotic formula for the $\Gamma$-function:

$$
\log \Gamma(u)=\left(u-\frac{1}{2}\right) \log u-u+\log \sqrt{2 \pi}+r(u), \quad|r(u)| \leqslant K|\operatorname{Re} u|^{-1},
$$

where $K$ is an absolute constant. Then we obtain

$$
\begin{aligned}
\prod_{j=0}^{s} \frac{\Gamma\left(t-b_{j}+1\right)}{\Gamma\left(t-a_{j}+1\right)}=\prod_{j=0}^{s} \frac{\Gamma\left(n\left(\tau-\beta_{j}\right)-1\right)}{\Gamma\left(n\left(\tau-\alpha_{j}\right)\right)} \\
=\exp \left\{\sum _ { j = 0 } ^ { s } \left(\left(\alpha_{j}-\beta_{j}\right) n \log n+n\left(\tau-\beta_{j}\right) \log \left(\tau-\beta_{j}\right)-n\left(\tau-\alpha_{j}\right) \log \left(\tau-\alpha_{j}\right)\right.\right. \\
\left.\left.\quad-\frac{3}{2} \log \left(\tau-\beta_{j}\right)+\frac{1}{2} \log \left(\tau-\alpha_{j}\right)-\frac{1}{2} \log n+O\left(n^{-1}\right)\right)\right\} .
\end{aligned}
$$

Finally, estimating the normalizing factor $\Pi(\boldsymbol{a}, \boldsymbol{b})$ in $(9)$ by $(21)$ we obtain expression (20) as required.

Lemma 7. Let $0<z<1, s=3$, the parameters $\boldsymbol{\alpha}, \boldsymbol{\beta}$ be defined in (13), and

$$
h(\tau)=z \prod_{j=0}^{s}\left(\tau+\alpha_{j}\right)-\prod_{j=0}^{s}\left(\tau+\beta_{j}\right)
$$

Suppose furthermore that

$$
h^{\prime}(0)<0, \quad h^{\prime \prime}(0)<0, \quad h^{\prime}\left(-\beta_{1}^{*}\right)<0, \quad \text { and } \quad h^{\prime \prime}\left(-\beta_{1}^{*}\right)>0 .
$$

Then the polynomial equation $h(\tau)=0$ has a unique positive root $\tau_{1}$ belonging to the interval $\left(0, z \alpha_{0}^{*} /(1-z)\right)$ and a unique root $\tau_{0}$ in the interval $\left(-\infty,-\beta_{1}^{*}\right)$.

Proof. A straightforward verification shows that in the case $s=3$ the second derivative $h^{\prime \prime}(\tau)$ is a quadratic polynomial with negative coefficients:

$$
h^{\prime \prime}(\tau)=12(z-1) \tau^{2}+6 \tau \sum_{j=0}^{3}\left(z \alpha_{j}-\beta_{j}\right)+2 \sum_{0 \leqslant j<k \leqslant 3}\left(z \alpha_{j} \alpha_{k}-\beta_{j} \beta_{k}\right) .
$$


In particular, $h^{\prime \prime}(\tau)<0$ for all $\tau \geqslant 0$. This means that the function $h^{\prime}(\tau)$ is strictly decreasing on $[0,+\infty)$ and, since $h^{\prime}(0)<0$, we have $h^{\prime}(\tau)<0$ on this interval. Whence $h(\tau)$ is strictly decreasing on $[0,+\infty)$ and, noting that $h(0)>0$ and $h(+\infty)=-\infty$, we deduce that the equation $h(\tau)=0$ has a unique positive root $\tau_{1}$. If $\tau \geqslant z \alpha_{0}^{*} /(1-z)$, then $z\left(\tau+\alpha_{0}^{*}\right) \leqslant \tau$ implies $h(\tau)<0$ by (13). Therefore, $\tau_{1} \in\left(0, z \alpha_{0}^{*} /(1-z)\right)$.

Consider now the interval $\left(-\infty,-\beta_{1}^{*}\right)$. First, note that the equation $h^{\prime \prime}(\tau)=$ 0 has two negative roots $\tau_{*}<\tau_{* *}<0$ by (23). Since $h^{\prime \prime}\left(-\beta_{1}^{*}\right)>0$, it follows that $-\beta_{1}^{*} \in\left(\tau_{*}, \tau_{* *}\right)$, therefore $h^{\prime \prime}(\tau)$ takes negative values on $\left(-\infty, \tau_{*}\right)$ and positive values on $\left(\tau_{*},-\beta_{1}^{*}\right)$. This means that the function $h^{\prime}(\tau)$ is strictly increasing on $\left(\tau_{*},-\beta_{1}^{*}\right)$. Taking into account that $h^{\prime}(-\infty)=+\infty, h^{\prime}\left(-\beta_{1}^{*}\right)<0, h(-\infty)=-\infty$, and $h\left(-\beta_{1}^{*}\right)>0$, we conclude that the equation $h(\tau)=0$ has a unique root $\tau_{0}$ in $\left(-\infty,-\beta_{1}^{*}\right)$, and the lemma is proved.

Lemma 8. Assume the conditions of Lemma 7 and, moreover, that $f^{\prime \prime}\left(-\tau_{0}\right) \neq 0$. Then the following asymptotic formulae are valid:

$$
\lim _{n \rightarrow \infty} \frac{\log |J(\boldsymbol{a}, \boldsymbol{b} ; z)|}{n}=f_{0}\left(\tau_{0}\right), \quad \lim _{n \rightarrow \infty} \frac{\log |I(\boldsymbol{a}, \boldsymbol{b}, z)|}{n}=\operatorname{Re} f_{0}\left(\tau_{1}\right)
$$

where

$$
\begin{aligned}
f_{0}(\tau)= & f(-\tau)+\tau \cdot f^{\prime}(-\tau) \\
= & \sum_{j=0}^{s}\left(\alpha_{j} \log \left(\tau+\alpha_{j}\right)-\beta_{j} \log \left(\tau+\beta_{j}\right)\right)+\sum_{j=1}^{s}\left(\beta_{j}-\alpha_{j}\right) \log \left(\beta_{j}-\alpha_{j}\right) \\
& \quad-\alpha_{0} \log \alpha_{0}+\alpha_{0}^{*} \log z .
\end{aligned}
$$

Proof. Note that $f^{\prime}(-\tau)=0$ implies $f(-\tau)=f_{0}(\tau)$.

The proof of the first equality is based on application of the saddle-point method to the integral representation of Lemma 6 . Take $\mu=-\tau_{0}$ in (20), where $\tau_{0}$ is defined in Lemma 7 . Since $\tau_{0} \in\left(-\infty,-\beta_{1}^{*}\right)$ is a zero of the polynomial (22) we see that

$$
z \prod_{j=0}^{s}\left(-\tau_{0}-\alpha_{j}\right)=\prod_{j=0}^{s}\left(-\tau_{0}-\beta_{j}\right)>0
$$

hence $f^{\prime}(\mu)=f^{\prime}\left(-\tau_{0}\right)=0$. Let us show that the function $\operatorname{Re} f(\mu+i v)$ of the real variable $v \in(-\infty,+\infty)$ attains its maximum at the unique point $v=0$. Indeed, we 
have

$$
\begin{aligned}
\frac{\mathrm{d}}{\mathrm{d} v} \operatorname{Re} f(\mu+i v)= & -\operatorname{Im} \frac{\mathrm{d}}{\mathrm{d} \tau} f(\mu+i v) \\
= & \sum_{j=0}^{1}\left(\arg \left(\mu+i v-\alpha_{j}^{*}\right)-\arg \left(\mu+i v-\beta_{j}\right)\right) \\
& \quad+\sum_{j=2}^{s}\left(\arg \left(\mu+i v-\alpha_{j}^{*}\right)-\arg \left(\mu+i v-\beta_{j}\right)\right) \\
= & \Sigma_{1}+\Sigma_{2},
\end{aligned}
$$

where $\beta_{0}=0$. Note that the function defined in (24) is odd with respect to the variable $v$. We assume that $v>0$ and consider the parametrization $\tau=\mu+i v=$ $\mu(1+i \tan \varphi)$ for $\varphi=\arg \tau \in(0, \pi / 2)$. Using the inequalities $0<\alpha_{0}^{*} \leqslant \alpha_{1}^{*}<\beta_{1} \leqslant$ $\beta_{1}^{*}<\mu$ we see that

$$
0<\arg \tau<\arg \left(\tau-\alpha_{0}^{*}\right) \leqslant \arg \left(\tau-\alpha_{1}^{*}\right)<\arg \left(\tau-\beta_{1}\right)<\frac{\pi}{2},
$$

hence

$$
0<\arg \left(\tau-\alpha_{0}^{*}\right)+\arg \left(\tau-\alpha_{1}^{*}\right)<\pi, \quad 0<\arg \tau+\arg \left(\tau-\beta_{1}\right)<\pi .
$$

Furthermore, we have

$$
\cot \arg \left(\tau-\alpha_{0}^{*}\right)=\frac{\mu-\alpha_{0}^{*}}{\mu \cdot \tan \varphi}, \quad \cot \arg \left(\tau-\alpha_{1}^{*}\right)=\frac{\mu-\alpha_{1}^{*}}{\mu \cdot \tan \varphi}
$$

denote

$$
\begin{aligned}
& C_{1}=\cot \left(\arg \left(\tau-\alpha_{0}^{*}\right)+\arg \left(\tau-\alpha_{1}^{*}\right)\right)=\frac{\mu^{2}\left(1-\tan ^{2} \varphi\right)-\mu\left(\alpha_{0}^{*}+\alpha_{1}^{*}\right)+\alpha_{0}^{*} \alpha_{1}^{*}}{\mu \cdot \tan \varphi \cdot\left(2 \mu-\alpha_{0}^{*}-\alpha_{1}^{*}\right)}, \\
& C_{2}=\cot \left(\arg \tau+\arg \left(\tau-\beta_{1}\right)\right)=\frac{\mu\left(1-\tan ^{2} \varphi\right)-\beta_{1}}{\left(2 \mu-\beta_{1}\right) \tan \varphi} .
\end{aligned}
$$

Then

$$
C_{1}-C_{2}=\frac{\mu^{2}\left(\beta_{1}-\alpha_{0}^{*}-\alpha_{1}^{*}\right)\left(1+\tan ^{2} \varphi\right)+\alpha_{0}^{*} \alpha_{1}^{*}\left(2 \mu-\beta_{1}\right)}{\mu\left(2 \mu-\alpha_{0}^{*}-\alpha_{1}^{*}\right)\left(2 \mu-\beta_{1}\right) \cdot \tan \varphi}>0
$$

by (13). Thus, $C_{1}>C_{2}$ or, equivalently,

$$
\arg \left(\tau-\alpha_{0}^{*}\right)+\arg \left(\tau-\alpha_{1}^{*}\right)<\arg \tau+\arg \left(\tau-\beta_{1}\right),
$$

i.e. $\Sigma_{1}<0$. If $v>0$, then in accordance with our choice of the parameters $\boldsymbol{\alpha}, \boldsymbol{\beta}$ given in (13),

$$
0<\arg \left(\mu-\alpha_{j}^{*}+i v\right)<\arg \left(\mu-\beta_{j}+i v\right)<\frac{\pi}{2}, \quad j=2, \ldots, s,
$$


yielding $\Sigma_{2}<0$. Finally, $\frac{\mathrm{d}}{\mathrm{d} v} \operatorname{Re} f(\mu+i v)=\Sigma_{1}+\Sigma_{2}<0$ for $v>0$ and $\frac{\mathrm{d}}{\mathrm{d} v} \operatorname{Re} f(\mu+$ $i v)>0$ for $v<0$ (by oddness of the derivative). Therefore, $\mu$ is the unique maximum of the function $\operatorname{Re} f(\tau)$ on the vertical line $(\mu-i \infty, \mu+i \infty)$. This implies that the integral $J$ is determined by the contribution of the saddle point $\mu$, namely,

$$
J(\boldsymbol{a}, \boldsymbol{b} ; z)=c_{3} g(\mu)\left|f^{\prime \prime}(\mu)\right|^{-1 / 2} e^{n f(\mu)}\left(1+O\left(n^{-1}\right)\right),
$$

whence the first equality of Lemma 8 follows.

In order to prove the second asymptotic relation, we write the sum $I$ in the form

$$
\begin{aligned}
I(\boldsymbol{a}, \boldsymbol{b} ; z) & =z^{a_{0}^{*}} \sum_{t=0}^{\infty} R(t) z^{t} \\
& =\frac{\prod_{j=1}^{s} \Gamma\left(\left(\beta_{j}-\alpha_{j}\right) n+1\right)}{\Gamma\left(\alpha_{0} n+1\right)} z^{\alpha_{0}^{*} n+1} \sum_{t=0}^{\infty} \prod_{j=0}^{s} \frac{\Gamma\left(t+\alpha_{j} n+1\right)}{\Gamma\left(t+\beta_{j} n+2\right)} z^{t}
\end{aligned}
$$

and use the techniques as in the second proof of Lemma 3 in [1]. We deduce that

$$
\begin{aligned}
\lim _{n \rightarrow \infty} & \frac{\log |I(\boldsymbol{a}, \boldsymbol{b}, z)|}{n} \\
= & \alpha_{0}^{*} \log z+\sum_{j=1}^{s}\left(\beta_{j}-\alpha_{j}\right) \log \left(\beta_{j}-\alpha_{j}\right)-\alpha_{0} \log \alpha_{0} \\
& \quad+\max _{\tau>0}\left\{\tau \log z+\sum_{j=0}^{s}\left(\left(\tau+\alpha_{j}\right) \log \left(\tau+\alpha_{j}\right)-\left(\tau+\beta_{j}\right) \log \left(\tau+\beta_{j}\right)\right)\right\} \\
= & \operatorname{Re} f_{0}\left(\tau_{1}\right)
\end{aligned}
$$

by Lemma 7 , thus completing the proof.

\section{Proof of the main result}

We shall apply the arithmetic and asymptotic results to the linear forms

$$
L(\boldsymbol{a}, \boldsymbol{b} ; z)=I(\boldsymbol{a}, \boldsymbol{b} ; z)-\operatorname{Li}_{1}(z) J(\boldsymbol{a}, \boldsymbol{b} ; z)=\sum_{j=2}^{s} P_{j}\left(z^{-1}\right) f_{j}(z)-P_{0}\left(z^{-1}\right)
$$

in the case $s=3$. It happens that an optimal choice for the integer parameters $\boldsymbol{\alpha}, \boldsymbol{\beta}$ depends on $z \in\{1 / 2,2 / 3,3 / 4,4 / 5\}$, but in all examples below the technical conditions of Lemmas 7 and 8 are fulfilled, and we also have $f_{0}\left(\tau_{0}\right) \neq \operatorname{Re} f_{0}\left(\tau_{1}\right)$ ensuring

$$
\lim _{n \rightarrow \infty} \frac{\log |L(\boldsymbol{a}, \boldsymbol{b} ; z)|}{n}=\max \left\{f_{0}\left(\tau_{0}\right), \operatorname{Re} f_{0}\left(\tau_{1}\right)\right\},
$$

where $\boldsymbol{a}$ and $\boldsymbol{b}$ are taken in accordance with (12). By Corollary of Lemma 3 the non-zero linear forms

$$
d^{\nu_{0} n} D_{\nu_{0} n} D_{\nu n}^{2} \cdot \Phi(\boldsymbol{a}, \boldsymbol{b})^{-1} \cdot L(\boldsymbol{a}, \boldsymbol{b} ; z), \quad d \text { is the denominator of } z^{-1},
$$


have integer coefficients; therefore, if $\nu_{0}(1+\log d)+2 \nu-\left(\int_{0}^{1} \varphi(x) \mathrm{d} \psi(x)-\int_{0}^{1 / \nu} \varphi(x) \frac{\mathrm{d} x}{x^{2}}\right)+\max \left\{f_{0}\left(\tau_{0}\right), \operatorname{Re} f_{0}\left(\tau_{1}\right)\right\}<0$, then these forms tend to 0 as $n \rightarrow \infty$, which implies that at least one of the numbers $f_{2}(z)$ and $f_{3}(z)$ must be irrational.

If $z=1 / 2$ we take

$$
\left(\alpha_{0}, \alpha_{1}, \alpha_{2}, \alpha_{3} ; \beta_{1}, \beta_{2}, \beta_{3}\right)=(3,4,5,6 ; 17,16,15) .
$$

Then

$$
\begin{aligned}
f_{0}\left(\tau_{0}\right) & =f_{0}(-54.774962 \ldots)=-30.978821 \ldots \\
\operatorname{Re} f_{0}\left(\tau_{1}\right) & =\operatorname{Re} f_{0}(0.045669 \ldots)=-31.342224 \ldots
\end{aligned}
$$

and

$\nu_{0}+2 \nu-\left(\int_{0}^{1} \varphi(x) \mathrm{d} \psi(x)-\int_{0}^{1 / \nu} \varphi(x) \frac{\mathrm{d} x}{x^{2}}\right)=14+2 \cdot 13-9.416718 \ldots=30.583281 \ldots$, since

$$
\varphi(x)=\left\{\begin{array}{cc}
2 \quad & \text { if } x \in\left[\frac{3}{13}, \frac{1}{4}\right) \cup\left[\frac{3}{11}, \frac{2}{7}\right) \cup\left[\frac{5}{9}, \frac{7}{12}\right) \cup\left[\frac{7}{11}, \frac{9}{14}\right) \cup\left[\frac{7}{9}, \frac{11}{14}\right) \cup\left[\frac{10}{11}, \frac{11}{12}\right), \\
1 \quad & \text { if } x \in\left[\frac{1}{13}, \frac{1}{10}\right) \cup\left[\frac{2}{13}, \frac{3}{13}\right) \cup\left[\frac{1}{4}, \frac{3}{11}\right) \cup\left[\frac{2}{7}, \frac{5}{12}\right) \cup\left[\frac{4}{9}, \frac{1}{2}\right) \cup\left[\frac{7}{13}, \frac{5}{9}\right) \\
& \cup\left[\frac{7}{12}, \frac{7}{11}\right) \cup\left[\frac{9}{14}, \frac{3}{4}\right) \cup\left[\frac{10}{13}, \frac{7}{9}\right) \cup\left[\frac{11}{14}, \frac{6}{7}\right) \cup\left[\frac{8}{9}, \frac{10}{11}\right) \cup\left[\frac{11}{12}, \frac{13}{14}\right), \\
0 & \text { otherwise. }
\end{array}\right.
$$

If $z=2 / 3$ we take

$$
\left(\alpha_{0}, \alpha_{1}, \alpha_{2}, \alpha_{3} ; \beta_{1}, \beta_{2}, \beta_{3}\right)=(5,6,7,8 ; 17,16,15) .
$$

Then

$$
\begin{aligned}
f_{0}\left(\tau_{0}\right) & =f_{0}(-67.615443 \ldots)=-32.714219 \ldots \\
\operatorname{Re} f_{0}\left(\tau_{1}\right) & =\operatorname{Re} f_{0}(0.314519 \ldots)=-34.100076 \ldots
\end{aligned}
$$

and

$$
\begin{aligned}
\nu_{0}(1 & +\log 2)+2 \nu-\left(\int_{0}^{1} \varphi(x) \mathrm{d} \psi(x)-\int_{0}^{1 / \nu} \varphi(x) \frac{\mathrm{d} x}{x^{2}}\right) \\
& =12 \cdot(1+\log 2)+2 \cdot 11-9.957743 \ldots=32.360022 \ldots,
\end{aligned}
$$

since

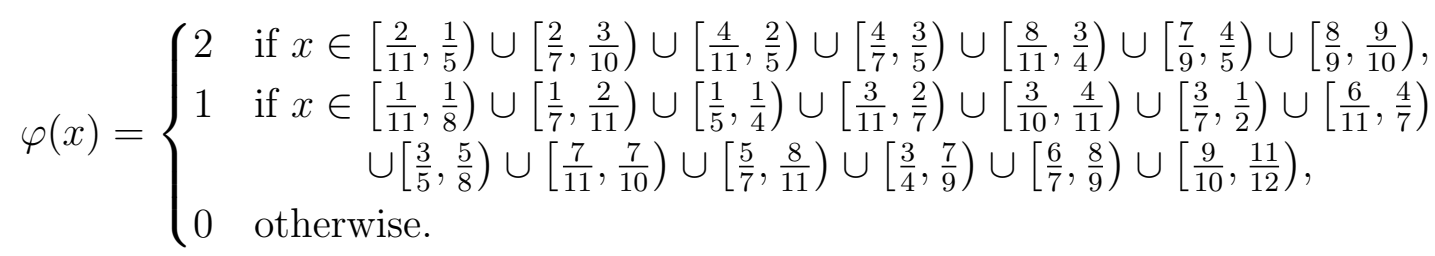


If $z=3 / 4$ we take

$$
\left(\alpha_{0}, \alpha_{1}, \alpha_{2}, \alpha_{3} ; \beta_{1}, \beta_{2}, \beta_{3}\right)=(7,8,9,10 ; 20,19,18) .
$$

Then

$$
\begin{aligned}
f_{0}\left(\tau_{0}\right) & =f_{0}(-96.814387 \ldots)=-42.031285 \ldots \\
\operatorname{Re} f_{0}\left(\tau_{1}\right) & =\operatorname{Re} f_{0}(0.679858 \ldots)=-40.372159 \ldots
\end{aligned}
$$

and

$$
\begin{aligned}
\nu_{0}(1+ & \log 3)+2 \nu-\left(\int_{0}^{1} \varphi(x) \mathrm{d} \psi(x)-\int_{0}^{1 / \nu} \varphi(x) \frac{\mathrm{d} x}{x^{2}}\right) \\
& =13 \cdot(1+\log 3)+2 \cdot 12-11.149689 \ldots=40.132270 \ldots
\end{aligned}
$$

since

$$
\varphi(x)=\left\{\begin{aligned}
& 2 \quad \text { if } x \in {\left[\frac{1}{4}, \frac{3}{11}\right) \cup\left[\frac{2}{5}, \frac{3}{7}\right) \cup\left[\frac{1}{2}, \frac{6}{11}\right) \cup\left[\frac{5}{8}, \frac{7}{11}\right) \cup\left[\frac{7}{10}, \frac{5}{7}\right) \cup\left[\frac{3}{4}, \frac{10}{13}\right) } \\
& \cup\left[\frac{4}{5}, \frac{9}{11}\right) \cup\left[\frac{9}{10}, \frac{10}{11}\right) \\
& 1 \quad \text { if } x \in {\left[\frac{1}{12}, \frac{1}{7}\right) \cup\left[\frac{1}{6}, \frac{1}{4}\right) \cup\left[\frac{3}{11}, \frac{4}{11}\right) \cup\left[\frac{3}{8}, \frac{2}{5}\right) \cup\left[\frac{3}{7}, \frac{5}{11}\right) \cup\left[\frac{6}{11}, \frac{4}{7}\right) } \\
& \cup\left[\frac{7}{12}, \frac{5}{8}\right) \cup\left[\frac{7}{11}, \frac{7}{10}\right) \cup\left[\frac{5}{7}, \frac{8}{11}\right) \cup\left[\frac{10}{13}, \frac{4}{5}\right) \cup\left[\frac{9}{11}, \frac{11}{13}\right) \\
& \cup\left[\frac{7}{8}, \frac{9}{10}\right) \cup\left[\frac{10}{11}, \frac{12}{13}\right)
\end{aligned}\right.
$$

If $z=4 / 5$ we take

$$
\left(\alpha_{0}, \alpha_{1}, \alpha_{2}, \alpha_{3} ; \beta_{1}, \beta_{2}, \beta_{3}\right)=(11,12,13,14 ; 26,25,24) .
$$

Then

$$
\begin{aligned}
f_{0}\left(\tau_{0}\right)=f_{0}(-136.248182 \ldots) & =-51.532175 \ldots \\
\operatorname{Re} f_{0}\left(\tau_{1}\right)=\operatorname{Re} f_{0}(1.686050 \ldots) & =-52.579778 \ldots
\end{aligned}
$$

and

$$
\begin{aligned}
\nu_{0}(1+ & \log 4)+2 \nu-\left(\int_{0}^{1} \varphi(x) \mathrm{d} \psi(x)-\int_{0}^{1 / \nu} \varphi(x) \frac{\mathrm{d} x}{x^{2}}\right) \\
& =15 \cdot(1+\log 4)+2 \cdot 14-12.384270 \ldots=51.410144 \ldots
\end{aligned}
$$

since

$$
\varphi(x)=\left\{\begin{aligned}
2 \quad \text { if } x \in & {\left[\frac{1}{14}, \frac{1}{13}\right) \cup\left[\frac{1}{12}, \frac{1}{11}\right) \cup\left[\frac{1}{7}, \frac{2}{13}\right) \cup\left[\frac{1}{6}, \frac{2}{11}\right) \cup\left[\frac{3}{14}, \frac{3}{13}\right) \cup\left[\frac{1}{4}, \frac{4}{15}\right) } \\
& \cup\left[\frac{3}{10}, \frac{4}{13}\right) \cup\left[\frac{5}{14}, \frac{4}{11}\right) \cup\left[\frac{3}{7}, \frac{5}{11}\right) \cup\left[\frac{1}{2}, \frac{7}{13}\right) \cup\left[\frac{3}{5}, \frac{8}{13}\right) \cup\left[\frac{5}{7}, \frac{8}{11}\right), \\
1 \quad \text { if } x \in & {\left[\frac{1}{13}, \frac{1}{12}\right) \cup\left[\frac{2}{13}, \frac{1}{6}\right) \cup\left[\frac{3}{13}, \frac{1}{4}\right) \cup\left[\frac{4}{15}, \frac{3}{11}\right) \cup\left[\frac{2}{7}, \frac{3}{10}\right) \cup\left[\frac{4}{13}, \frac{5}{14}\right) } \\
& \cup\left[\frac{4}{11}, \frac{5}{13}\right) \cup\left[\frac{2}{5}, \frac{3}{7}\right) \cup\left[\frac{5}{11}, \frac{6}{13}\right) \cup\left[\frac{7}{13}, \frac{6}{11}\right) \cup\left[\frac{4}{7}, \frac{3}{5}\right) \cup\left[\frac{8}{13}, \frac{7}{11}\right) \\
& \cup\left[\frac{9}{14}, \frac{5}{7}\right) \cup\left[\frac{8}{11}, \frac{13}{15}\right) \cup\left[\frac{9}{10}, \frac{14}{15}\right)
\end{aligned}\right.
$$

The presented computations show the truth of Theorem 1. 


\section{References}

[1] K. Ball and T. Rivoal (2001): Irrationalité d'une infinité de valeurs de la fonction zêta aux entiers impairs, Invent. Math. 146:1, 193-207.

[2] S. Fischler and T. Rivoal (2003): Approximants de Padé et séries hypergéométriques équilibrées, J. Math. Pures Appl. 82:10, 1369-1394.

[3] L. A. Gutnik (1983): Irrationality of some quantities that contain $\zeta(3)$, Acta Arith. 42:3, 255-264. (Russian)

[4] T. G. Hessami Pilehrood (1999): On the linear independence of vectors with polylogarithmic coordinates, Moscow Univ. Math. Bull. 54:6, 40-42.

[5] L. Lewin (1981): Polylogarithms and associated functions, Elsevier North Holland, New York.

[6] G. Rhin and C. Viola (1996): On a permutation group related to $\zeta(2)$, Acta Arith. 77:1, 23-56.

[7] G. Rhin and C. Viola (2001): The group structure for $\zeta(3)$, Acta Arith. 97:3, 269-293.

[8] G. Rhin and C. Viola (2005): The permutation group method for the dilogarithm, Ann. Scuola Norm. Sup. Pisa (5) 4, 389-437.

[9] W. Zudilin (2004): Arithmetic of linear forms involving odd zeta values, $J$. Théorie Nombres Bordeaux 16:1, 251-291.

Kh. Hessami Pilehrood and T. Hessami Pilehrood:

Mathematics Department,

Shahrekord University,

P.O. Box 115, Shahrekord, IRAN

School of Mathematics,

Institute for Studies in Theoretical Physics and Mathematics,

P.O. Box 19395-5746, Tehran, IRAN

E-mail: hessamik@ipm.ir, hessamit@ipm.ir

W. Zudilin:

Department of Mechanics and Mathematics,

Moscow Lomonosov State University,

Vorobiovy Gory, GSP-2,

119992 Moscow, RUSSIA

E-mail: wadim@ips.ras.ru 